\title{
Heterointerface Engineering of Broken-Gap InAs/GaSb Multilayer Structures
}

\author{
Jheng-Sin Liu, Yan Zhu, Patrick S. Goley, and Mantu K. Hudait* \\ Advanced Devices \& Sustainable Energy Laboratory (ADSEL), Bradley Department of Electrical and Computer Engineering, Virginia \\ Tech, Blacksburg, Virginia 24061, United States
}

ABSTRACT: Broken-gap InAs/GaSb strain balanced multilayer structures were grown by molecular beam epitaxy (MBE), and their structural, morphological, and band alignment properties were analyzed. Precise shutter sequence during the MBE growth process, enable to achieve the strain balanced structure. Cross-sectional transmission electron microscopy exhibited sharp heterointerfaces, and the lattice line extended from the top GaSb layer to the bottom InAs layer. X-ray analysis further confirmed a strain balanced InAs/ $\mathrm{GaSb}$ multilayer structure. A smooth surface morphology with surface roughness of $\sim 0.5 \mathrm{~nm}$ was demonstrated. The effective barrier height $-0.15 \mathrm{eV}$ at the $\mathrm{GaSb} / \mathrm{InAs}$ heterointerface was determined by X-ray photoelectron spectroscopy, and it was further corroborated by simulation. These results are important to demonstrate desirable characteristics of mixed $\mathrm{As} / \mathrm{Sb}$ material systems for high-performance and low-power tunnel field-effect transistor applications.

KEYWORDS: broken gap, tunnel transistors, InAs/GaSb, molecular beam epitaxy, heterointerface

\section{INTRODUCTION}

Scaling down metal-oxide-semiconductor field-effect transistors below $10 \mathrm{~nm}$ and high power consumption at OFF-state are important issues for a nanoscale silicon transistor. Low supply voltage operation and steep switching characteristics are essential for a next-generation low-power nanoscale transistor. Tunnel field-effect transistors (TFETs) with sub-60 mV/dec subthreshold swing (SS) based on the band-to-band tunneling mechanism at room temperature can help to realize low OFFstate leakage at low supply voltage of operation. Very recently, mixed arsenide/antimonide based $\operatorname{In}_{x} \mathrm{Ga}_{1-x} \mathrm{As} / \mathrm{GaAs}_{1-y} \mathrm{Sb}_{y}$ TFET device structures with variable arsenic (As) and antimony $(\mathrm{Sb})$ alloy compositions for a wide range of adjustable effective tunnel barrier height $\left(E_{\text {beff }}\right)$, such that these two layers are internally lattice matched, were extensively studied by several researchers. ${ }^{1-3}$ In such configuration, controlling alloy compositions while simultaneously maintaining the internally lattice matched condition is extremely challenging due to the formation of an unwanted layer by surface termination of atoms at the source/channel heterointerface. However, mixed $\mathrm{As} / \mathrm{Sb}$ based heterostructures provide attractive band gaps and tunable band alignment for a p-type TFET with $\operatorname{In}_{x} \mathrm{Ga}_{1-x} \mathrm{As} / \mathrm{GaAs}_{1-y} \mathrm{Sb}_{y}$ as a source/channel configuration. Furthermore, a p-type InAs/GaSb TFET exhibited an $\mathrm{ON}$ current of $I_{\mathrm{ON}}=85 \mu \mathrm{A} / \mu \mathrm{m},{ }^{2}$ and the same structure in $\mathrm{n}$-channel configuration revealed an $\mathrm{ON}$ current of $I_{\mathrm{ON}}=180 \mu \mathrm{A} / \mu \mathrm{m}$ at $V_{\mathrm{GS}}=V_{\mathrm{DS}}=0.5 \mathrm{~V}$ with $I_{\mathrm{ON}} / I_{\mathrm{OFF}}$ ratio of 6 $\times 10^{3}{ }^{3}$ In this InAs/GaSb TFET configuration, the ideal band alignment would be a broken gap configuration. However, the

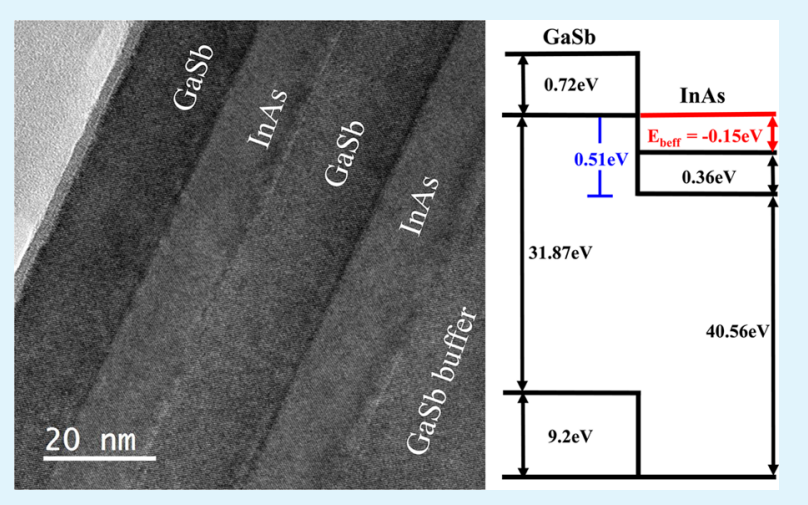

local strain at the InAs/GaSb interface depends on two types of interfacial bonds: tensile $\mathrm{Ga}-\mathrm{As}$ bonds and compressive $\mathrm{In}-\mathrm{Sb}$ bonds. ${ }^{4}$ As a result of these interfacial bonds, the interface could be GaAs-like due to $\mathrm{Ga} / \mathrm{In}$ exchange during the growth of an InAs/GaSb TFET structure. Moreover, different types of interfacial layers have distinct strain relaxation properties and one (GaAs-like) will introduce large number of dislocations at the $0.62 \%$ tensile strained InAs on GaSb substrate that will increase the OFF-state current in an InAs/GaSb TFET. $^{5}$ On the other hand, the formation of InSb-like interfacial layer at the InAs/GaSb heterointerface would compensate the tensile strain, and an additional compressive strain amount will help to decrease the defect density and prevent strain relaxation. As a result, one could achieve strain balanced InAs/GaSb multilayer heterostructures by controlling proper thicknesses of each layer and switching sequences of $\mathrm{As}, \mathrm{Sb}, \mathrm{In}$, and $\mathrm{Ga}$ at each heterointerface during growth. However, measures must be taken during the molecular beam epitaxy (MBE) growth of mixed As/Sb based heterostructures due to a strong competition between different incorporation rates of anions (As and $\mathrm{Sb}$ ) due to their dissimilar sticking coefficients and the metallic character of $\mathrm{Sb}$.

This study focuses on the growth, structural, and morphological properties, strain relaxation, band alignment, and simulated $E_{\mathrm{beff}}$ of the strain balanced InAs/GaSb multilayer

Received: October 24, 2014

Accepted: January 8, 2015 

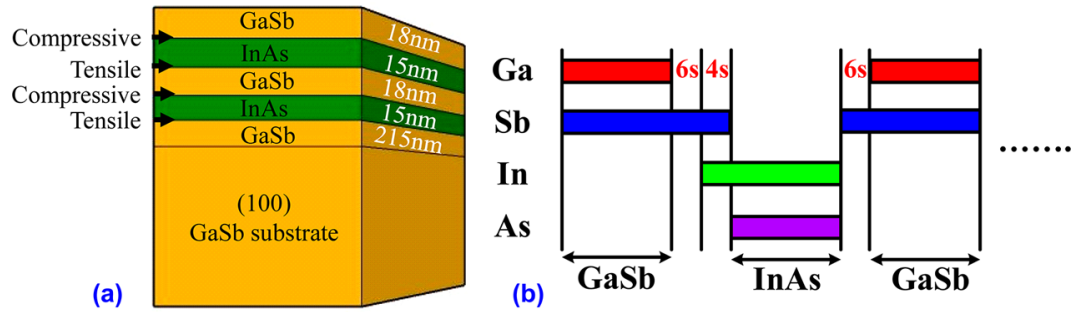

Figure 1. (a) Schematic diagram of InAs/GaSb multilayer heterostructure and (b) MBE shutter sequence for this multilayer growth with InSb deposition at the $\mathrm{GaSb} / \mathrm{InAs}$ and antimony soaking at the InAs/GaSb heterointerface. The layer InAs/GaSb substrate interface is tensile, and the $\mathrm{GaSb} / \mathrm{InAs}$ interface is compressive, as indicated.

structure grown by solid source MBE. The strain compensation was achieved by carefully controlling the shutter sequences of growth species at each heterointerface and proper thicknesses of each layer during growth. The strain relaxation properties of $\mathrm{InAs} / \mathrm{GaSb}$ heterostructures was evaluated using high-resolution X-ray diffraction. Cross-sectional transmission electron microscopy (TEM) was used to investigate the defect properties and surface morphology was analyzed using atomic force microscopy (AFM). The X-ray photoelectron spectroscopy (XPS) measurement was carried out to determine the band alignment at the InAs/GaSb heterointerface and the experimental band offset values were further confirmed by modeling.

\section{RESULTS AND DISCUSSION}

Material Characterization. Figure 1a shows the schematic of the InAs/GaSb multilayer heterostructure selected for this work, where the thickness of each layer is indicated in this figure, and Figure $1 \mathrm{~b}$ shows the shutter sequences for this growth. The nominal thickness of each layer is about $15 \mathrm{~nm}$, and the lattice mismatch between the InAs and $\mathrm{GaSb}$ is about $0.62 \%$. To achieve well-controlled coherent heterointerface, GaAs-like interfacial layer must be prevented during growth. In the shutter sequence, two methods were used to form InSb-like interfacial layer: (1) InSb stacking and (2) Sb soaking. After the completion of $\mathrm{GaSb}$ layer growth, the $\mathrm{Sb}$ flux was left open for $6 \mathrm{~s}$ followed by $4 \mathrm{~s}$ of indium (In) exposure to create an artificial thin InSb layer in between the $\mathrm{GaSb}$ and InAs layer. The As flux was open for the duration of the InAs layer growth, and prior to the GaSb deposition, the InAs surface was soaked by $\mathrm{Sb}$ for $6 \mathrm{~s}$. Because the vapor pressure of As is higher than that of $\mathrm{Sb}$, the exposure of $\mathrm{Sb}$ on the surface of InAs for a short duration helps prevent As from escaping the surface of InAs prior to GaSb layer growth. Thus, through the subtle control of the InAs/GaSb epitaxial heterointerface, we could achieve the overall strain balanced structure, and both the InAs and GaSb layers are internally lattice matched in the growth sequence studied here. The InAs/GaSb substrate interface is tensile (T) and $\mathrm{GaSb} / \mathrm{InAs}$ interface is compressive $(\mathrm{C})$ as indicated in Figure 1a, and the equal number of $\mathrm{C} / \mathrm{T}$ layers balances the strain, which can be seen from the cross-sectional TEM as well as X-ray analysis below.

The InAs/GaSb multilayer heterostructure was investigated by cross-sectional TEM measurement and analysis. Figure $2 \mathrm{a}, \mathrm{b}$ shows the high-resolution TEM micrographs of the InAs/GaSb multilayer stack consisting of 4 layer ( 2 tensile interface and 2 compressive interface). In this figure, each layer and interface were labeled to match with the growth sequence of the multilayer stack, as shown in Figure 1a. Figure 2c,d shows highresolution lattice indexing of the epitaxial InAs layer on $\mathrm{GaSb}$

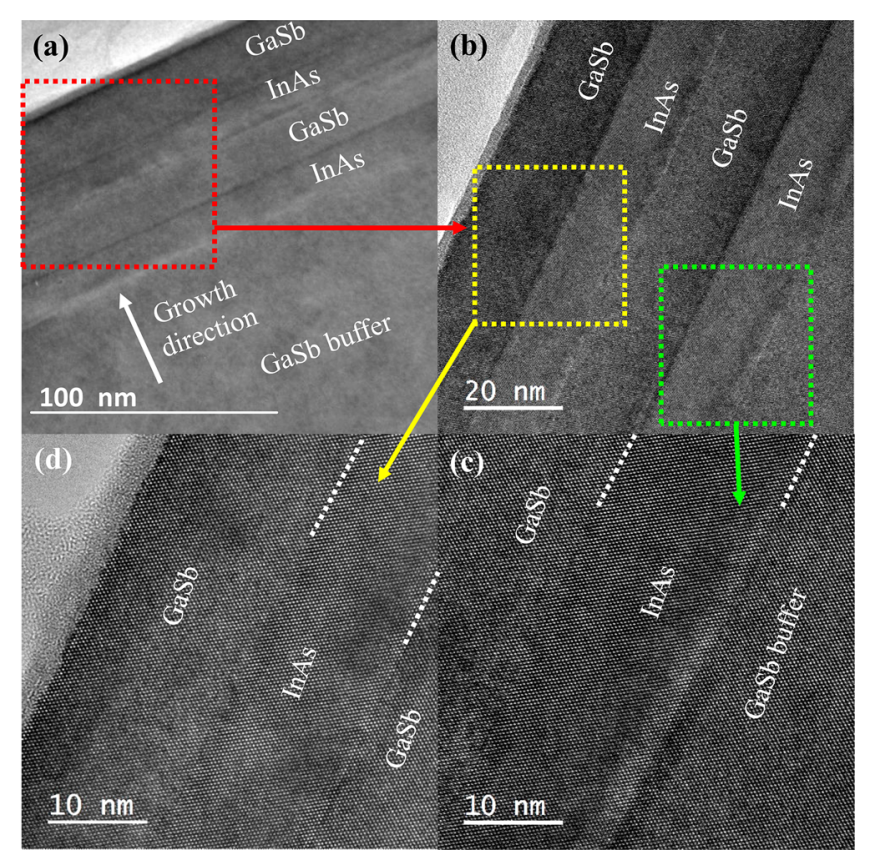

Figure 2. (a) High-resolution cross-sectional TEM micrograph of InAs/GaSb multilayer structure and $(\mathrm{b}-\mathrm{d})$ lattice indexing of the $\mathrm{GaSb} / \mathrm{InAs} / \mathrm{GaSb}$ stack showing each abrupt heterointerface.

buffer layer and epitaxial GaSb on InAs epilayer, respectively. These TEM micrographs showed that the epilayers were homogeneous and with smooth heterointerfaces. In the InAson-GaSb interface, an intentionally added $4 \mathrm{~s}$ of growth time enabled the $\mathrm{InSb}$ interfacial layer to achieve a high-quality heterointerface, and this layer will compensate the tensile strain amount of $0.62 \%$ between InAs and GaSb. The mixture fluxes of both In and $\mathrm{Sb}$ limited the As coverage, which tends to form $\mathrm{GaAsSb}$, thus accumulating more tensile strain at the InAs-onGaSb heterointerface. ${ }^{4,6}$ Furthermore, Sb soaking after the InAs deposition also restricted As coverage and prevented the formation of a GaAs-like layer. In fact, the exposure of $\mathrm{Sb}$ on the surface of InAs prior to $\mathrm{GaSb}$ growth prevents the As from escaping the InAs surface and acts as a surfactant during growth. Both methods tailored to create InSb-like interfacial layer at each heterointerface. The InSb-like interfaces provide strain compensation and virtually defect-free heterointerfaces. Each interface exhibited an abrupt heterojunction, which is essential for high-performance TFET structure that can reduce the leakage current under reverse gate voltage.

High-resolution X-ray diffraction was used to investigate the strain relaxation properties of $\mathrm{InAs} / \mathrm{GaSb}$ multilayer layer structure. Figure 3 shows X-ray rocking curve of $18 \mathrm{~nm} \mathrm{GaSb} /$ $15 \mathrm{~nm}$ InAs/18 nm GaSb/15 nm InAs/GaSb substrate 


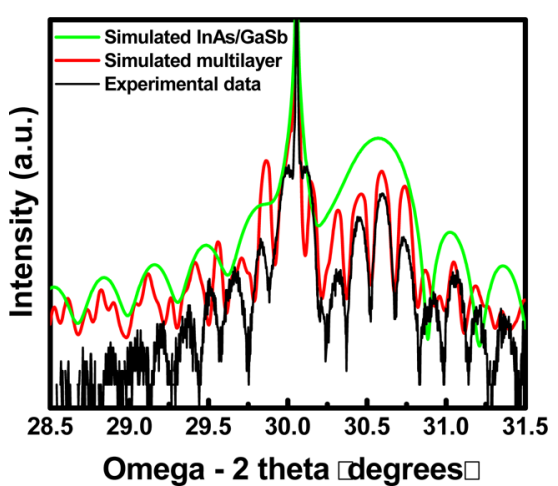

Figure 3. (Black) Symmetric experimental (004) rocking curve and (red) simulated rocking curve of InAs/GaSb multilayer structure, and (green) simulated rocking curve of only $15 \mathrm{~nm}$ InAs on $\mathrm{GaSb}$ with an ultrathin $0.5 \mathrm{~nm}$ layer of InSb. The thickness fringes demonstrate the superior quality of this heterostructure. The envelope line of three secondary fringes were InAs epilayers affected by multilayer structures.

structure. A simulation was performed to identify the position of the InAs peak from thickness fringes. In Figure 3, a green line shows the simulated rocking curve from $15 \mathrm{~nm}$ InAs layer on $\mathrm{GaSb}$ substrate with the addition of an ultrathin $0.5 \mathrm{~nm} \mathrm{InSb}$ interfacial layer. One can locate the InAs peak position with respect to the $\mathrm{GaSb}$ substrate peak. However, the simulated rocking curve with $0.5 \mathrm{~nm} \mathrm{InSb}$ interfacial layer at InAs on $\mathrm{GaSb}$ and $\mathrm{GaSb}$ on InAs heterointerface exhibited that the InAs layer was affected in the multilayer configuration. Besides, InAs layers provide an envelope function for fringes next to the $\mathrm{GaSb}$ peak and have a peak-pulling effect due to the multilayer structures. The simulated rocking curve also shows the complexity of fringes due to InAs/GaSb multilayer structure with an InSb interfacial layer. The simulation further confirms the existence of interfacial layers, shown in Figure $2 b-d$. Figure 4 shows the symmetric (004) and asymmetric (115) reciprocal
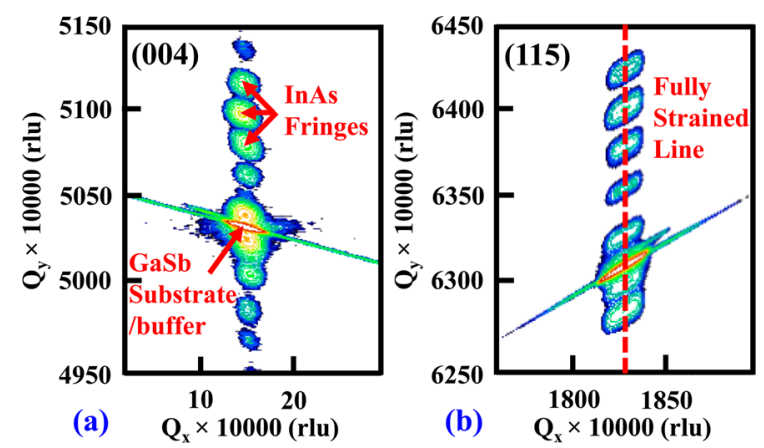

Figure 4. (a) Symmetric (004) and (b) asymmetric (115) reciprocal space maps of InAs/GaSb multilayer structure. The thickness fringes become contour of intensity. All the thickness fringes of InAs and $\mathrm{GaSb}$ RLPs lie along the fully strained vertical line.

space maps (RSMs) of the layer structure, respectively. As Figure 4a shows, the thickness fringes become the contours of intensity, and the InAs reciprocal lattice point (RLP) is in agreement with the X-ray rocking curve. The stronger intensity of the InAs RLP (shown by an arrow) provided the information on the layer separate from the thickness fringes. Moreover, the RLP of the GaSb substrate and the InAs is in the same vertical line indicates minimal or no lattice tilt, as expected because the entire layer is strain balanced. Further, the properties of relaxation and strain state could be obtained from asymmetric (115) RSM, shown in Figure 4b. One can find that all of the RLPs are lying on the fully strained line, which further confirms the balanced strain generated by compressively strained GaSbon-InAs and tensile strained InAs-on-GaSb by proper heterointerface engineering. The aligned vertical line indicates the same in-plane lattice constant of InAs and $\mathrm{GaSb}$, supported by the TEM results discussed above. One can anticipate that a GaAs-like interfacial layer might abolish the strain balance system by introducing additional tensile strain in the structure. ${ }^{6}$ An intentionally inserted ultrathin InSb interfacial layer at the bottom interface and an InAsSb layer due to $\mathrm{As} / \mathrm{Sb}$ exchange balanced the strain in interface, thus creating a strain balanced structure. Thus, an abrupt heterointerface and well-controlled strain compensation are suitable for further high-performance $\mathrm{n}$ - and p-channel TFET applications.

The layer structure was further characterized using AFM. Figure 5 shows the surface morphology from the surfaces of 2

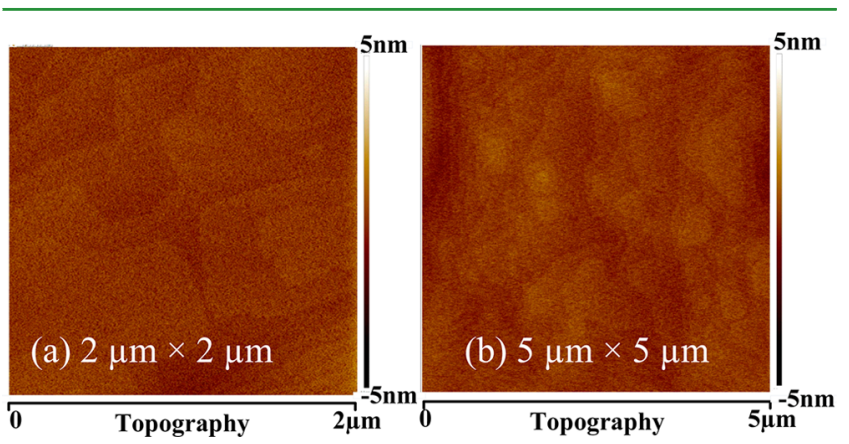

Figure 5. Surface morphology of multilayer structures were investigated by AFM. The root-mean-square (rms) surface roughness of 0.42 and $0.55 \mathrm{~nm}$ were recorded from (a) $2 \times 2 \mu \mathrm{m}$ and (b) $5 \times 5$ $\mu \mathrm{m}$ area scan, respectively.

$\times 2 \mu \mathrm{m}$ and $5 \times 5 \mu \mathrm{m}$ area scans. The surface root-mean-square (rms) roughness were found to be $\sim 0.42$ and $0.55 \mathrm{~nm}$, respectively. Smooth surface morphology implies minimal dislocations inside the epitaxial layer structure, which is supported by both TEM and X-ray analysis above. Furthermore, lower surface roughness can also be related to well-controlled elements switching during growth. In fact, lower rms surface roughness was reported by creating an ultrathin layer of InAs-like interface during the growth of InGaAs/ GaAsSb TFET structure. ${ }^{5}$

Band Offset. The band offset parameters at the InAs/GaSb heterointerface are an important design parameter for a broken gap InAs/GaSb based tunnel FET structure. X-ray photoelectron spectroscopy spectra revealed binding energy information for each materials. The core levels (CLs) and valence band maxima (VBM) spectra were recorded to determine the band alignment between the $\mathrm{GaSb}$ and the InAs layer. The band alignment properties were determined by Kraut's method. ${ }^{7}$ The valence band offset (VBO) between the $\mathrm{GaSb}$ and the InAs can be expressed as

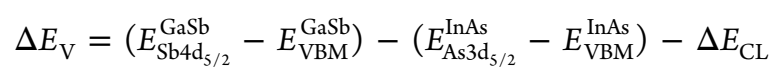

where $E_{\mathrm{Sb} 4 \mathrm{~d}_{5 / 2}}^{\mathrm{GaSb}}$ and $E_{\mathrm{As} 3 \mathrm{~d}_{5 / 2}}^{\mathrm{InAs}}$ are CL binding energy of $\mathrm{Sb} 4 \mathrm{~d}_{5 / 2}$ and As $3 \mathrm{~d}_{5 / 2}$ from $\mathrm{GaSb}$ and InAs layer, respectively; $E_{\mathrm{VBM}}^{\mathrm{GaSb}}$ and $E_{\mathrm{VBM}}^{\mathrm{InAs}}$ were VBMs for the respective materials. $\Delta E_{\mathrm{CL}}=E_{\mathrm{Sb} 4 \mathrm{~d}_{5 / 2}}^{\mathrm{GaSb}}-$ $E_{\mathrm{As} 3 \mathrm{~d}_{5 / 2}}^{\mathrm{InAs}}$ is the difference in CL binding energy from the $\mathrm{GaSb}$ 
(a)
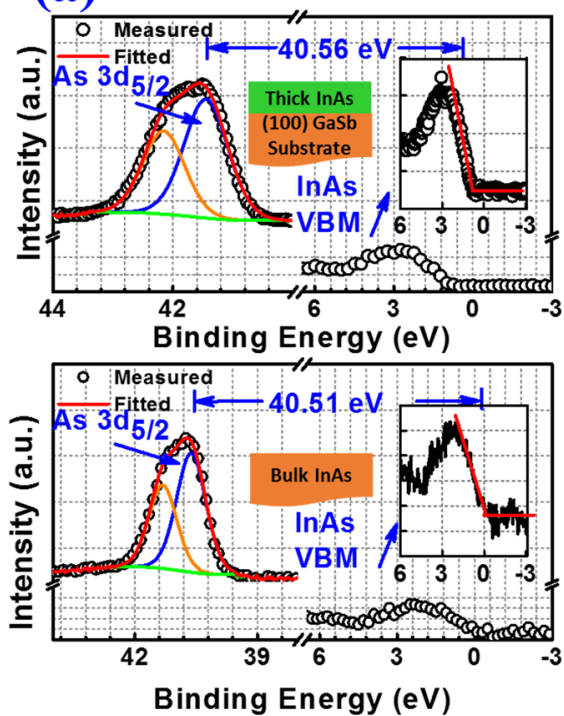

(c)

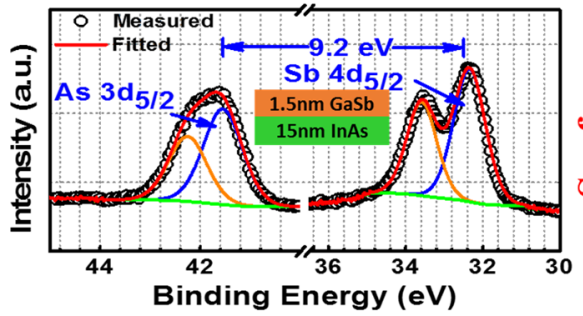

(b)
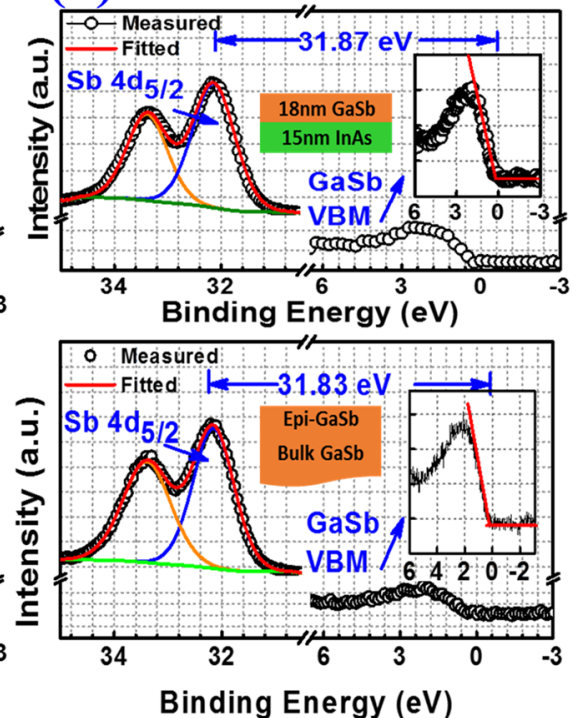

(d)

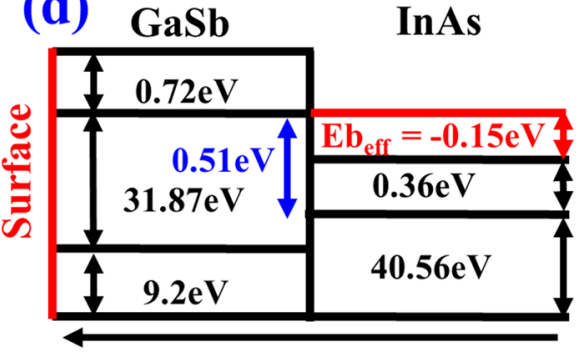

Growth direction

Figure 6. XPS spectra of (a) As $3 \mathrm{~d}$ core level $\left(E_{\mathrm{As}}^{\mathrm{As}}\right.$ d $)$ and valence band maximum VBM $\left(E_{\mathrm{VBM}}^{\mathrm{InAs}}\right)$ from thick InAs film on GaSb substrate and bulk InAs, (b) Sb 4d ( $\left.E_{S b 4 d}^{\mathrm{Sb}}\right)$ core level and VBM ( $\left.E_{\mathrm{VBM}}^{\mathrm{GaSb}}\right)$ from thick GaSb film on multilayer structure and $1 \mu \mathrm{m}$ epi-GaSb on GaSb substrate, (c) As 3d, $\mathrm{Sb} 4 \mathrm{~d}$ core levels from $\sim 1.5 \mathrm{~nm} \mathrm{GaSb} / \mathrm{InAs}$ interface, and (d) energy-band alignment of the $\mathrm{GaSb} / \mathrm{InAs}$ heterointerface, respectively.

and InAs heterointerface. Figure 6 shows the CL binding energies and corresponding VBMs from the thick InAs layer, thick GaSb layer, and the $\mathrm{GaSb} / \mathrm{InAs}$ heterointerface. Using the measured data and the equation above, the $\mathrm{VBO}$ was determined to be $0.51 \pm 0.05 \mathrm{eV}$. A constant flow of electrons was used throughout the XPS measurements to compensate the charging effect. Using the bandgap values of InAs $\left(E_{G}^{\text {InAs }}=0.36\right.$ $\mathrm{eV})$ and $\mathrm{GaSb}\left(E_{G}^{\mathrm{GaSb}}=0.72 \mathrm{eV}\right)$ at $300 \mathrm{~K}$, the effective barrier height, $E_{\text {beff }}=E_{G}^{\text {InAs }}-\Delta E_{V}$ of $-0.15 \mathrm{eV}$ was determined, as shown in Figure 6d. It is worth nothing that the band bending effect caused by the semiconductor-to-semimetal transition should be considered during determining the band alignment especially in the broken gap system..$^{8-10}$ To address this issue, we have measured XPS spectra from the bulk InAs and homoepitaxial $1 \mu \mathrm{m}$ thick epi-GaSb on $\mathrm{GaSb}$ substrate. The energy difference between the CL and the VBM for a thick 20 $\mathrm{nm}$ epi-InAs and the bulk InAs substrate are 40.56 and 40.51 $\mathrm{eV}$, respectively, as shown in Figure 6a. Similarly, the energy differences are 31.87 and $31.83 \mathrm{eV}$ for $18 \mathrm{~nm} \mathrm{GaSb}$ and $1 \mu \mathrm{m}$ thick epi-GaSb on GaSb substrate, respectively, shown in Figure $6 \mathrm{~b}$. One can find from Figure $6 \mathrm{a}, \mathrm{b}$ that the difference (CL to VBM) between the epilayer and the bulk are 0.05 and $0.04 \mathrm{eV}$ for InAs and $\mathrm{GaSb}$, respectively. These values are within the XPS measurement error bar. ${ }^{11}$ This $E_{\text {beff }}$ value is in agreement with the previously reported results. ${ }^{12-14}$ Here, the negative value indicates the broken gap configuration at the $\mathrm{GaSb} / \mathrm{InAs}$ heterointerface. This $E_{\text {beff }}$ value determines the ON- and OFF- state current of a mixed $\mathrm{As} / \mathrm{Sb}$ based tunnel FET device structure.

To correlate the experimental band alignment values and hence the $E_{\text {beff }}$ as a function of In and $\mathrm{Sb}$ alloy compositions in InGaAs/GaAsSb TFET structure, we performed the modeling using TCAD Sentaurus, version $\mathrm{H}$. Figure $7 \mathrm{a}$ shows both simulated and experimental $E_{\text {beff }}$ corresponding to a various In and $\mathrm{Sb}$ compositions in $\mathrm{In}_{x} \mathrm{Ga}_{1-x} \mathrm{As} / \mathrm{GaAs}_{1-y} \mathrm{Sb}_{y}$ system such that for each In and $\mathrm{Sb}$ composition, the InGaAs and $\mathrm{GaAsSb}$ layers are internally lattice matched. Simulation model was calibrated with the experimental band alignment value with the In composition of $x=0.7$ and the $\mathrm{Sb}$ composition of $y=0.65$. $^{15}$ One can find from this Figure $7 \mathrm{a}$ that the $E_{\text {beff }}$ value decreases from $0.44 \mathrm{eV}$ where $x=0.53$ and $y=0.49$ (staggered gap) to $-0.1 \mathrm{eV}$ where $x=1$ and $y=1$ (broken gap) with increasing In and $\mathrm{Sb}$ compositions. The lowest simulated staggered gap $E_{\text {beff }}$ is $0.06 \mathrm{eV}$ for an $\mathrm{In}_{0.9} \mathrm{Ga}_{0.1} \mathrm{As} / \mathrm{GaAs}_{0.1} \mathrm{Sb}_{0.9}$ TFET structure. The results of simulated $I_{\mathrm{ON}}$ as a function of $E_{\text {beff }}$ are shown in Figure $7 \mathrm{~b}$. The inset shows the drain current, $I_{D}$, with gate voltage swing. To improve the ability of gate control in a nanoscale TFET, the double gate $\mathrm{p}-\mathrm{i}-\mathrm{n}$ TFET (channel thickness of $5 \mathrm{~nm}$ ), long channel (channel length of $40 \mathrm{~nm}$ ), and low equivalent oxide thickness (EOT of $1 \mathrm{~nm}$ ) were used in this simulation. Asymmetric doping of source and drain values of $4 \times 10^{19} \mathrm{~cm}^{-3}$ and $6 \times 10^{17} \mathrm{~cm}^{-3}$, respectively, were considered to prevent ambipolar current conduction. It was found that the $I_{\mathrm{ON}}$ current is strongly dependent on the $E_{\text {beff }}$ under $V_{\mathrm{GS}}=V_{\mathrm{DS}}=0.5 \mathrm{~V}$ conditions. The increase in $I_{\mathrm{ON}}$ in this 

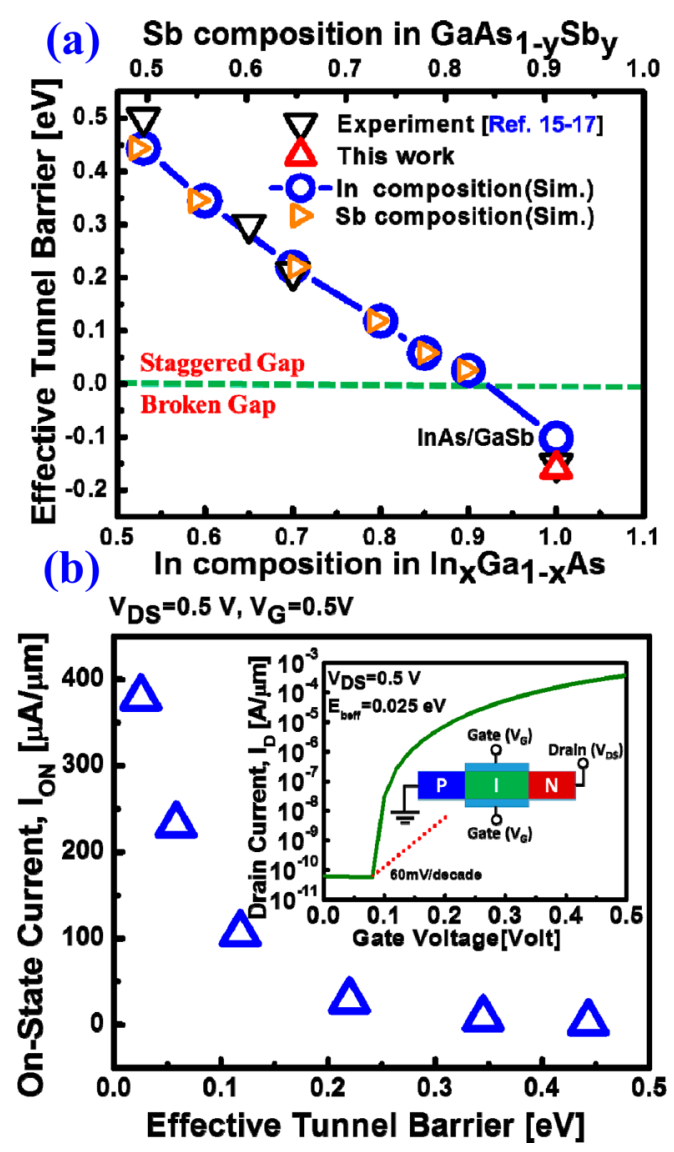

Figure 7. (a) Experimental and simulated $E_{\text {beff }}$ as a function of In and $\mathrm{Sb}$ compositions in $\mathrm{GaAs}_{1-y} \mathrm{Sb}_{y} / \mathrm{In}_{x} \mathrm{Ga}_{1-x} \mathrm{As}$ material system. The experimental results are in agreement with the simulated prediction. (b) Simulated $I_{\mathrm{ON}}$ as a function of effective tunnel barrier height.

material system is believed to be due to the increase in tunneling probability compared with the material system of $0.35 \mathrm{eV}$ tunnel barrier height. However, compositions lower than $\mathrm{In}_{0.9} \mathrm{Ga}_{0.1} \mathrm{As} / \mathrm{GaAs}_{0.1} \mathrm{Sb}_{0.9}$ system, the $E_{\text {beff }}$ is negative, and even higher ON current can be attainable, but measures must be taken to reduce the OFF-state leakage of such structure. The quantization and strain effect were not taken into account in this calculation. This first-order $E_{\text {beff }}$ calculation, which is in agreement with the experimental results, ${ }^{15-17}$ provides critical guidance for designing mixed As/Sb-based tailor-made tunnel FET device structure.

\section{CONCLUSIONS}

In summary, high-quality broken gap strain balanced InAs/ $\mathrm{GaSb}$ multilayer structure, grown using solid source MBE, with InSb-like interfacial layer were demonstrated by analyzing structural, morphological, and band alignment properties. Cross-sectional TEM micrographs exhibited sharp heterointerfaces and lattice lines extended from the top GaSb layer to the bottom InAs layer. X-ray analysis further confirmed a strain balanced InAs/GaSb multilayer structure. A smooth surface morphology with surface roughness of about $0.5 \mathrm{~nm}$ was achieved. The effective barrier height at the GaSb/InAs heterointerface was determined to be $-0.15 \mathrm{eV}$ by $\mathrm{X}$-ray photoelectron spectroscopy, which is in agreement with other reported results. This was further corroborated by simulation. These results are important to demonstrate desirable characteristics of mixed $\mathrm{As} / \mathrm{Sb}$ material systems for superlattice and tunnel FET applications. Thus, one can tailor the energy barrier of internally lattice matched GaAsSb/InGaAs heterointerface by precisely controlling the growth sequences of antimony- and arsenide-based material systems.

\section{MATERIALS AND METHODS}

Material Synthesis. The periodic InAs and GaSb layers were grown by solid source MBE on (100) GaSb substrate with $215 \mathrm{~nm}$ undoped $\mathrm{GaSb}$ buffer layer at $550{ }^{\circ} \mathrm{C}$ with $\mathrm{As} / \mathrm{In}=20, \mathrm{Sb} / \mathrm{Ga}=5$ and a growth rate of $\sim 0.2 \mu \mathrm{m} / \mathrm{h}$ and $\sim 0.43 \mu \mathrm{m} / \mathrm{h}$, respectively. The GaSb substrate oxide desorption was performed under $\mathrm{Sb}$ flux at $600{ }^{\circ} \mathrm{C}$. A low-temperature $\mathrm{Sb}$ effusion cell was used for the $\mathrm{Sb}$ flux during growth. The surface pattern was constantly monitored by reflection high-energy electron diffraction and during the entire layer structure growth. The thickness of each layer and the shutter sequences were carefully selected such that the InAs/GaSb material system is internally strain balanced. This was performed by creating an ultrathin layer of $\mathrm{InSb}(\mathrm{As})$ in between the InAs and the $\mathrm{GaSb}$.

Materials Characterization. To determine the structural quality and the relaxation state of InAs/GaSb multilayer stack, we recorded high-resolution triple axis X-ray rocking curve. Cross-sectional highresolution transmission electron microscopy (HR-TEM) was used to characterize the heterointerface between the InAs and the $\mathrm{GaSb}$ as well as $\mathrm{GaSb}$ and InAs epilayer. HR-TEM imaging was performed using JEOL 2100 transmission electron microscope. For this purpose, the electron transparent foils of thin film cross-section of InAs/GaSb were prepared by standard polishing technique (i.e., mechanical grinding, dimpling and $\mathrm{Ar}^{+}$ion beam milling). The band alignment of InAs/ GaSb heterointerface was investigated using a PHI Quantera SXM XPS system with a monochromated $\mathrm{Al} \mathrm{K} \alpha$ (energy of $1486.7 \mathrm{eV}$ ) Xray source. The As $3 \mathrm{~d}_{5 / 2}$ and $\mathrm{Sb} 4 \mathrm{~d}_{5 / 2}$ CL binding energy spectra as well as InAs and $\mathrm{GaSb}$ valence band binding energy spectra were collected with a pass energy of $26 \mathrm{eV}$ and an exit angle of $45^{\circ}$. The binding energy was corrected by adjusting the carbon (C) 1s CL peak position to $285.0 \mathrm{eV}$ for each sample surface. Curve fitting was performed by the CasaXPS 2.3.14 using a Lorentzian convolution with a Shirley-type background. The CL energy position was defined to be the center of the peak width at the half of the peak height. The VBM values were determined by linear extrapolation of the leading edge to the baseline of the valence band spectra. The VBM value is sensitive to the choice of points on the leading edge used to obtain the regression line. The uncertainty of $\Delta E_{\mathrm{v}}$ value was found to be in the range of \pm 0.05 in the present work by the regressions analysis of selected data over the linear region.

\section{AUTHOR INFORMATION}

\section{Corresponding Author}

*Tel: (540) 231-6663. Fax: (540) 231-3362. E-mail: mantu. hudait@vt.edu.

\section{Notes}

The authors declare no competing financial interest.

\section{ACKNOWLEDGMENTS}

This work is supported in part by National Science Foundation (NSF) under Grant No. ECCS-1028494 and Intel Corporation. J.-S.L. and Y.Z. acknowledge support from NSF under Grant No. ECCS-1348653 and ECCS-1028494, respectively. P.G. acknowledges support from the NSF Graduate Research Fellowship under Grant No. DGE 0822220. The authors would like to acknowledge N. Jain and M. Clavel for AFM and $\mathrm{X}$-ray measurements. The authors would also like to acknowledge the NCFL-Institute for Critical Technology and Applied Sciences (ICTAS) and Virginia Tech Nanofabrication Facilities for materials characterization. 


\section{REFERENCES}

(1) Zhu, Y.; Hudait, M. K. Low-Power Tunnel Field-Effect Transistors Using Mixed $\mathrm{As}$ and $\mathrm{Sb}$ Based Heterostructures. Nanotechnol. Rev. 2013, 2, 637-678.

(2) Rajamohanan, B.; Mohata, D.; Zhu, Y.; Hudait, M.; Jiang, Z.; Hollander, M.; Klimeck, G.; Datta, S. Design, Fabrication, and Analysis of p-Channel Arsenide/Antimonide Hetero-Junction Tunnel Transistors. J. Appl. Phys. 2014, 115, 044502-1-044502-7.

(3) Guangle, Z.; Li, R.; Vasen, T.; Qi, M.; Chae, S.; Lu, Y.; Zhang, Q.; Zhu, H.; Kuo, J. M.; Kosel, T.; Wistey, M.; Fay, P.; Seabaugh, A.; Xing, H. Gate-Recessed Vertical InAs/GaSb TFETs with Record High $I_{\mathrm{ON}}$ of $180 \mu \mathrm{A} / \mu \mathrm{m}$ at $V_{\mathrm{DS}}=0.5 \mathrm{~V}$. IEEE Int. Electron Devices Meet. 2012, $777-780$.

(4) Mahalingam, K.; Haugan, H. J.; Brown, G. J.; Aronow, A. J. Strain Analysis of Compositionally Tailored Interfaces in InAs/GaSb Superlattices. Appl. Phys. Lett. 2013, 103, 211605-1-211605-4.

(5) Zhu, Y.; Jain, N.; Vijayaraghavan, S.; Mohata, D. K.; Datta, S.; Lubyshev, D.; Fastenau, J. M.; Liu, W. K.; Monsegue, N.; Hudait, M. K. Role of InAs and GaAs Terminated Heterointerfaces at Source/ Channel on the Mixed As-Sb Staggered Gap Tunnel Field-Effect Transistor Structures Grown by Molecular Beam Epitaxy. J. Appl. Phys. 2012, 112, 024306-1-024306-16.

(6) Huang, Y.; Ryou, J.-H.; Dupuis, R. D.; Zuo, D.; Kesler, B.; Chuang, S.-L.; Hu, H.; Kim, K.-H.; Lu, Y. T.; Hsieh, K. C.; Zuo, J.-M. Strain-Balanced InAs/GaSb Type-II Superlattice Structures and Photodiodes Grown on InAs Substrates by Metalorganic Chemical Vapor Deposition. Appl. Phys. Lett. 2011, 99, 011109-1-011109-3.

(7) Kraut, E. A.; Grant, R. W.; Waldrop, J. R.; Kowalczyk, S. P. Semiconductor Core-Level to Valence-Band Maximum BindingEnergy Differences: Precise Determination by X-ray Photoelectron Spectroscopy. Phys. Rev. B 1983, 28, 1965-1977.

(8) Sai-Halasz, G. A.; Esaki, L.; Harrison, W. A. InAs-GaSb Superlattice Energy Structure and Its Semiconductor-Semimetal Transition. Phys. Rev. B 1978, 18, 2812-2818.

(9) Altarelli, M. Electronic Structure and Semiconductor-Semimetal Transition in InAs-GaSb Superlattices. Phys. Rev. B 1983, 28, 842845.

(10) Lapushkin, I.; Zakharova, A.; Yen, S. T.; Chao, K. A A Selfconsistent Investigation of the Semimetal-Semiconductor Transition in InAs/GaSb Quantum Wells under External Electric Fields. J. Phys.: Condens. Matter 2004, 16, 4677-4684.

(11) Hudait, M. K.; Zhu, Y.; Maurya, D.; Priya, S.; Patra, P. K.; Ma, A.W. K.; Aphale, A.; Macwan, I. Structural and Band Alignment Properties of $\mathrm{Al}_{2} \mathrm{O}_{3}$ on Epitaxial Ge Grown on (100), (110) and (111)A GaAs Substrates by Molecular Beam Epitaxy. J. Appl. Phys. 2013, 113, 134311-1-134311-8.

(12) Gualtieri, G. J.; Nuzzo, R. G.; Malik, R. J.; Walker, J. F.; Feldman, L. C.; Sunder, W. A.; Schwartz, G. P. Summary Abstract: Valence-Band Discontinuities in (100) GaSb/AlSb and GaSb/InAs Heterojunctions. J. Vac. Sci. Technol. B 1987, 5, 1284-1285.

(13) Kroemer, H. The $6.1 \AA$ Family (InAs, GaSb, AlSb) and its Heterostructures: A Selective Review. Phys. E 2004, 20, 196-203.

(14) Vurgaftman, I.; Meyer, J. R.; Ram-Mohan, L. R. Band Parameters for III-V Compound Semiconductors and Their Alloys. J. Appl. Phys. 2001, 89, 5815-5875.

(15) Zhu, Y.; Jain, N.; Vijayaraghavan, S.; Mohata, D. K.; Datta, S.; Lubyshev, D.; Fastenau, J. M.; Liu, A. K.; Monsegue, N.; Hudait, M. K. Defect Assistant Band Alignment Transition from Staggered to Broken Gap in Mixed As/Sb Tunnel Field-Effect Transistor Heterostructure. J. Appl. Phys. 2012, 112, 094312-1-094312-9.

(16) Mohata, D.; Rajamohanan, B.; Mayer, T.; Hudait, M.; Fastenau, J.; Lubyshev, D.; Liu, A. W. K.; Datta, S. Barrier-Engineered ArsenideAntimonide Heterojunction Tunnel FETs With Enhanced Drive Current. IEEE Int. Electron Devices Meet. 2012, 33, 1568-1570.

(17) Sai-Halasz, G. A.; Chang, L. L.; Welter, J. M.; Chang, C. A.; Esaki, L. Optical Absorption of $\mathrm{In}_{1-x} \mathrm{Ga}_{x} \mathrm{As} / \mathrm{GaSb}_{1-y} \mathrm{As}_{y}$ Superlattices. Solid State Commun. 1978, 27, 935-937. 